\title{
MORPHOLOGY AND THERMAL BEHAVIOUR OF POLY(METHYL METHACRYLATE)/POLY(ETHYLENE GLYCOL) SEMI-INTERPENETRATING POLYMER NETWORKS
}

\author{
SHANGGUAN LIN-JIAN ${ }^{1}$, LIU GUOQIN ${ }^{2, I^{*}}$ \\ ${ }^{1}$ School of Mechanical Engineering, North China University of Water Conservancy and Electric Power, Zhengzhou 450011, China \\ ${ }^{2}$ College of Material Science and Engineering, Henan University of Technology, Zhengzhou, 450001, China.
}

(Received: April 8, 2011 - Accepted: June 21, 2011)

\begin{abstract}
Poly(methyl methacrylate) (PMMA) networks and linear poly(ethylene glycol) (PEG) can form semi-interpenetrating polymer networks (semi-IPNs), i.e. PMMA/PEG semi-IPNs. The morphology and thermal behaviors of PMMA/PEG semi-IPNs was studied by DSC, TEM, Optical Microscopy, and SEM. The results indicated that the content of PEG and the cross-linking density of PMMA networks have an important influence on the phase separation in the PMMA/PEG semi-IPNs. When the content of PEG and cross-linking density is more than 35 and $3.5 \mathrm{wt} \%$, respectively, PMMA/PEG semi-IPNs are a mixture of PEG in the crystalline state with PMMA networks in the amorphous state.
\end{abstract}

Keywords: poly (methyl methacrylate), poly (ethylene glycol), semi-IPNs, morphology, thermal behavour.

\section{INTRODUCTION}

Due to van der Waals type bonding between the PMMA chains and the planar poly (ethylene glycol) (PEG) segments ${ }^{1,2}$, Linear poly(methyl methacrylate) (PMMA) can form stable blends with linear PEG. The bonding is the non-covalent association between groups on different polymer chains ${ }^{3}$. These macromolecular blends form under conditions in which the polymers are thermodynamically compatible.

However, it is reported that the miscible domain formed at the content of 10 and $30 \mathrm{wt} \%$ of PEG by weight; It was indicated that this blend is probably immiscible with PEG $>30 \mathrm{wt} \%$ and crystalline aggregates of PEG can be observed ${ }^{4,5}$.

Interpenetrating polymer networks (IPNs), which are composed of a hydrophobic and hydrophilic component are defined as a combination of two or more polymers in network form, at least one of which is polymerized and/ or crosslinked in the immediate presence of the other(s $)^{6}$. Interpenetrating polymerization represents an innovative approach to solving the problem of polymer incompatibility ${ }^{7-9}$.

There are a variety of combinations possible with IPNs resulting in a broad range of properties. The performance of IPNs is determined by the physical and chemical nature of the constitutive networks, the relative proportion of components, the physical interactions between constitutive networks, the IPNs preparation procedure, and so on ${ }^{10-16}$, which were proved to show potential applicability for biomedical materials, membranes for separation, or selective absorbents ${ }^{17-19}$. Besides full IPNs, in which both components are crosslinked independently of each other, there are also other types of IPNs such as semiIPNs $^{20-22}$. Semi-IPNs have only one component cross-linked and can be used biocompatible materials ${ }^{22}$

In our previous paper, we synthesized blends of poly((methacrylic acid)co-(methyl methacrylate)) (P(MAA-co-MMA) networks with poly(ethylene glycol) $(\mathrm{PEG})^{23}$, and poly(acrylic acid-co-butyl methacrylate) (P(AA-coBMA)) networks with poly(N-vinyl-2-pyrrolidone) (PVP) ${ }^{24}$, and P(MMA-coVP)/PEG semi-IPNs ${ }^{25}$, which are stabilized by hydrogen bonds.

In this paper, we present the morphology and thermal behaviour of poly(methyl methacrylate) /poly(ethylene glycol) (PMMA/PEG) semi-IPNs in details. Investigation of the specific features of the PMMA/PEG semi-IPNs is of substantial practical interest because of their applications as shape memory materials ${ }^{23}$.

\section{EXPERIMENTAL}

\section{Materials}

Methyl methacrylate (MMA) and azobisisobutyronitrile (AIBN) were of analytical grade obtained from the Chengdu Reagent Factory. Ethylene glycol dimethacrylate (EGDMA) was purchased from Aldrich Chemical Co (Aldrich). MMA was distilled under reduced pressure before use. AIBN, used as a radical initiator, was recrystallized from methanol solution. EGDMA was used as a cross-linker without further purification. PEG (Aldrich) with catalogue number-average molecular weights of 1000, 2000, 4000, 6000, 10000 and 20000 was dried by heating at $70^{\circ} \mathrm{C}$ for $7 \mathrm{hr}$ under vacuum.

Preparation of the PMMA/PEG semi-IPNs

Radical polymerization of 50.5-60.5 wt $\%$ MMA was carried out at $55^{\circ} \mathrm{C}$ for 24 hours under a nitrogen atmosphere in the presence of 35-45 wt $\%$ linear PEG. AIBN (0.5 wt $\%$ with respect to monomers) and EGDMA (0.6-5.8 wt $\%$ with respect to monomers) was employed as an initiator and a crosslinker, respectively. The experimental design is summarized in Table 1. The prepared polymer are quenched, and then dried under vacuum at room temperature for 10 days to remove unreacted monomers. According to the formulations of PEG content and compositions of PMMA/PEG semi-IPNs, these polymers were represented as A (molecular weight of PEG) series, B (content of PEG) series and $\mathrm{C}$ (cross-linking density of network) series.

\section{Differential Scanning Calorimetry (DSC) measurements}

The glass-transition temperature $(T)$ and melting temperature $(T)$ of the polymer was measured using a DSC from DuPont (DSC-9900). The samples were kept at $170^{\circ} \mathrm{C}$ for $3 \mathrm{~min}$ and then were cooled quickly to $-70^{\circ} \mathrm{C}$ from the melt of the first scan. The $\mathrm{T}_{\mathrm{g}}$ was obtained at the inflection point of the jump heat capacity using a scan rate of $10^{\circ} \mathrm{C} / \mathrm{min}$ within the temperature range from $-70^{\circ} \mathrm{C}$ to $170^{\circ} \mathrm{C}$. All measurements were conducted under a nitrogen atmosphere. The cell was calibrated using an indium standard; the weight of the sample was 5-10 mg. 
Table 1 Information on PMMA/PEG semi-IPNs and $\mathrm{T}_{\mathrm{g}}$ in this study.

\begin{tabular}{|c|c|c|c|c|}
\hline \multirow{2}{*}{ Notation } & \multirow{2}{*}{$\begin{array}{c}\text { Cross-linking } \\
\text { density (wt \%) }\end{array}$} & \multicolumn{2}{|c|}{ PEG } & \multirow{2}{*}{$T_{g}$} \\
\cline { 3 - 4 } & MW & C (wt \%) & $(\square)$ \\
\hline \multicolumn{2}{|c|}{ A (Molecular weight of PEG) series } & & \\
\hline A1 & 3.5 & 1000 & 38 & 66 \\
\hline A2 & 3.5 & 2000 & 38 & 80 \\
\hline A3 & 3.5 & 4000 & 38 & 83 \\
\hline A4 & 3.5 & 6000 & 38 & 86 \\
\hline A5 & 3.5 & 10000 & 38 & 88 \\
\hline A6 & 3.5 & 20000 & 38 & 93 \\
\hline B (content of PEG) series & & & \\
\hline B1 & 3.5 & 4000 & 35 & 95 \\
\hline B2 (A3) & 3.5 & 4000 & 38 & 83 \\
\hline B3 & 3.5 & 4000 & 40 & 78 \\
\hline B4 & 3.5 & 4000 & 45 & 72 \\
\hline C (Cross-linking density of network) series & & \\
\hline C1 & 0.6 & 2000 & 38 & 77 \\
\hline C2 & 1.2 & 2000 & 38 & 78 \\
\hline C3 (A2) & 3.5 & 2000 & 38 & 80 \\
\hline C4 & 5.8 & 2000 & 38 & 84 \\
\hline
\end{tabular}

Optical microscopy (OM) observations

The spherulitic morphologies was observed and photographed through a polarizing microscope (Jiangnan XPR-2, China) equipped with a digital camera and a heating stage.

Transmission electron microscope (TEM) observations

TEM measurements were carried out on a JEM-100CX Microscopy (Japan Electronic Company). Samples were prepared by dropping a suspension onto Formvar-coated copper grids.

Scanning electron microscope (SEM) observations

The fracture surface morphology of the PMMA/PEG semi-IPNs was observed in a scanning electron microscope (model AMRAY 1000) after being sputter-coated with gold.

\section{RESULTS AND DISCUSSION}

PMMA networks were prepared by radical homopolymerization in the presence of linear PEG. So, the PMMA/PEG is a semi-interpenetrating polymer networks (semi-IPNs), i.e., crosslinked PMMA networks and linear PEG. It is known that PMMA networks prepared by radical polymerization are amorphous and PEG is a semicrystalline polymer. Because the proportion of PEG exceeds $35 \mathrm{wt} \%$ in this study, the PMMA/PEG semi-IPNs produces a semicrystalline mixture, namely that the two-component system is expected to be composed of amorphous blended regions with some crystalline regions made up entirely of PEG, which is a shape memory polymer and exhibits independently two shape memory effects ${ }^{26}$.

DSC is extensively used to investigate miscibility in polymer blends or complexes. A single compositionally dependent glass transition is an indication of full miscibility at a dimensional scale between 20 and $40 \mathrm{~nm}^{27}$. It would be of interest to study the effects of chain length of PEG on the complexation with the PMMA networks. Figure 1 shows the DSC scanning curves of the PMMA networks and A series. The pure amorphous PMMA network exhibits one $\mathrm{T}_{\sigma}$ at $110 \square$, while the addition of PEG to PMMA networks causes depression of the $T_{g}$ of the semi-IPNs, the smaller the molecular weight of PEG, the more the decreased $T_{g}$. PMMA/PEG semi-IPNs display a single $T_{q}$, which strongly suggests the amorphous phase of two components are miscible because the attractive forces exists between the negatively charged oxygen atoms of PEG and positively charged carbonyl carbon atoms of PMMA.

In further experiments, we have also studied the influence of the PEG content on PMMA/PEG semi-IPNs. As shown in Figure 2, in the case of $T$ of the semi-IPNs, it decreases with increasing of PEG content (see Table 1). At the same time, endothermic peaks due to the melting of PEG crystallites can been observed, and $T_{m} \mathrm{~s}$ of PEG in semi-IPNs somewhat increase with increasing of PEG content (see Table 2). It is believed that more addition of PEG to PMMA networks not only produces an increase in crystal content and but also introduces more amorphous PEG into semi-IPNs, because PEG is a semicrystalline polymer; the former effect can increase the crystallinity of semi-IPNs, and, according to a qualitative analysis of the Fox equation, the latter decreases $T_{g}$ of polymer. More crystal content can not mean distinct increases of $T_{m}$, because $T_{m}$ is mainly related to crystal size, the surface free energy in the PEG crystals, and so on.

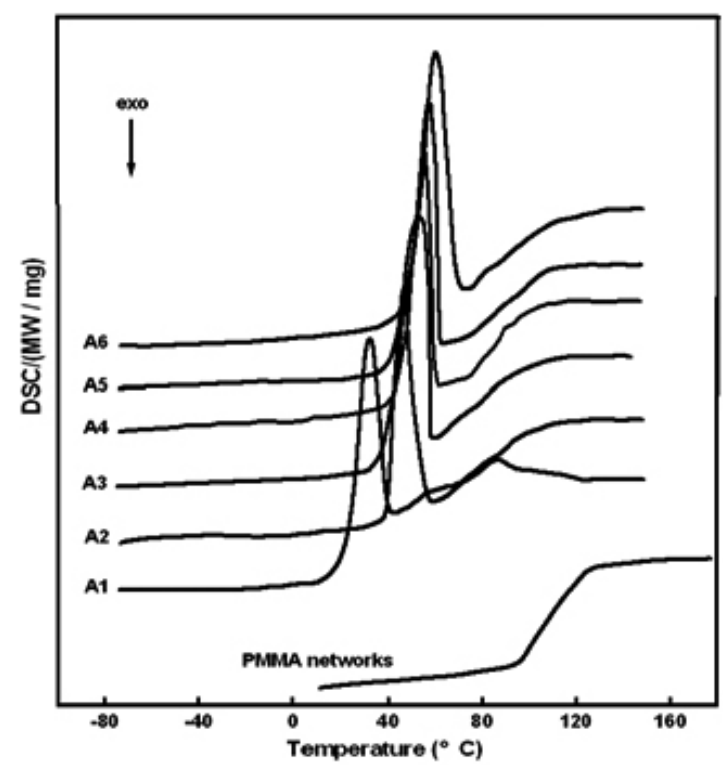

Figure 1. DSC scans of PMMA networks and A series.

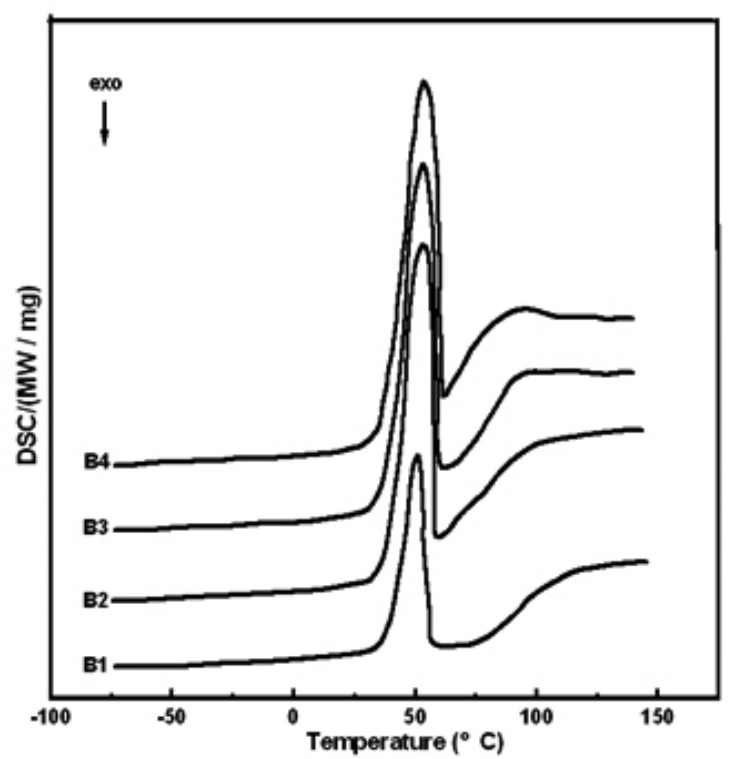

Figure 2. DSC scans of B series. 


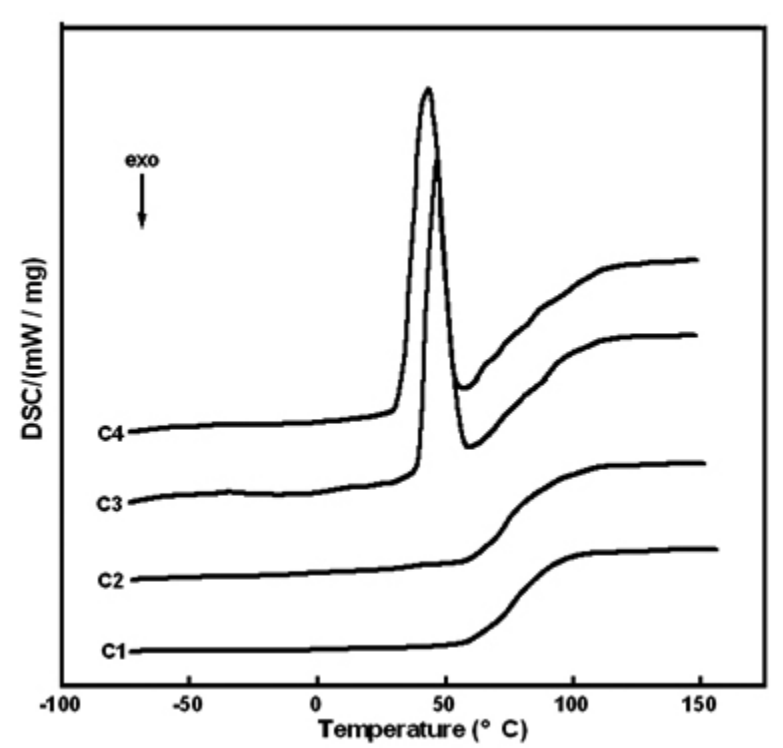

Figure 3. DSC scans of C series.

Table 2. $T_{m} \mathrm{~s}$ of PMMA/PEG semi-IPNs.

\begin{tabular}{|c|c|c|c|}
\hline Sample & $T_{m}\left({ }^{\circ} \mathrm{C}\right)$ & Sample & $T_{m}\left({ }^{\circ} \mathrm{C}\right)$ \\
\hline A1 & 32.3 & B2 (A3) & 52.8 \\
\hline A2 & 46.8 & B3 & 52.9 \\
\hline A3 & 52.8 & B4 & 53.6 \\
\hline A4 & 55.4 & C1 & - \\
\hline A5 & 57.4 & C2 & - \\
\hline A6 & 60.2 & $\mathrm{C} 3(\mathrm{~A} 2)$ & 46.8 \\
\hline B1 & 51.2 & $\mathrm{C} 4$ & 43.91 \\
\hline
\end{tabular}

The influence of cross-linking density on the thermal behavior of PMMA/ PEG semi-IPNs is presented in Figure 3. It is worth noting that cross-linking density plays an important role for phase separation of semi-IPNs. When the weight fraction of the cross-linker is less than $3.5 \mathrm{wt} \%$, the PMMA/PEG semiIPNs shows only one single glass transition temperature and the endothermic peaks due to the melting of PEG crystallites wasn't observed, suggesting that IPNs are fully miscible with a homogeneous amorphous phase. While the weight fraction of the cross-linker is more than $7 \mathrm{wt} \%$, the polymer is very fragile and unsuitable for being materials. When the cross-linker content is between 3.5-7wt \%, the distinct endothermic peak of PEG crystal fusion can be observed, indicating that the phase separations occur in the seim-IPNs. Furthermore, with increasing of cross-linking density in semi-IPNs, $T_{m}$ of PEG decreases. These results may be interpreted as follows: on the one hand, the Flory-Huggins interaction $\left(\chi_{12}\right)$ decreases with the increasing of the crosslinking density ${ }^{28}$, which results from decreases of free volume and gives an unfavourable contribution to the miscibility; on the other hand, higher crosslinking density (corresponding to more network nodes) of PMMA network would reduce crystal perfectness and crystal size of PEG by the restricted chain mobility, as evidenced by the later optical microscopy (OM) observation, which causes a $\mathrm{T}_{\mathrm{m}}$ depression.
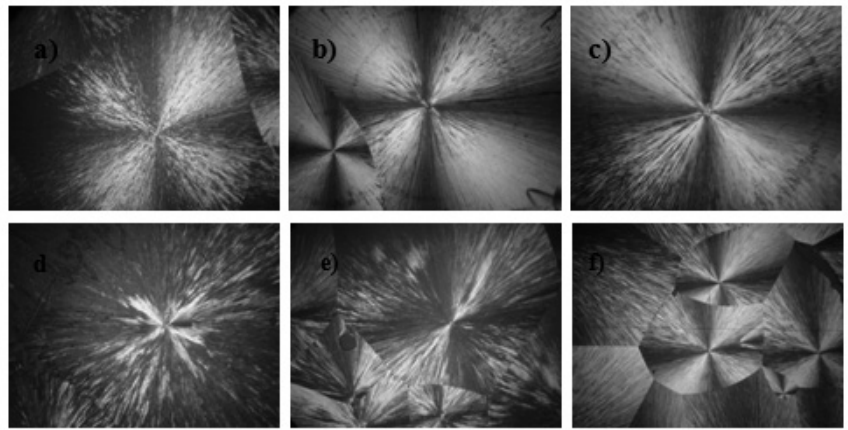

Figure 4. Optical micrographs (crossed polars: $\times 20$ ) of PEG spherulites with various molecular weight of PEG (a: 1000; b: 2000; c: 4000; d: 6000; e: 10000; f: 20000).
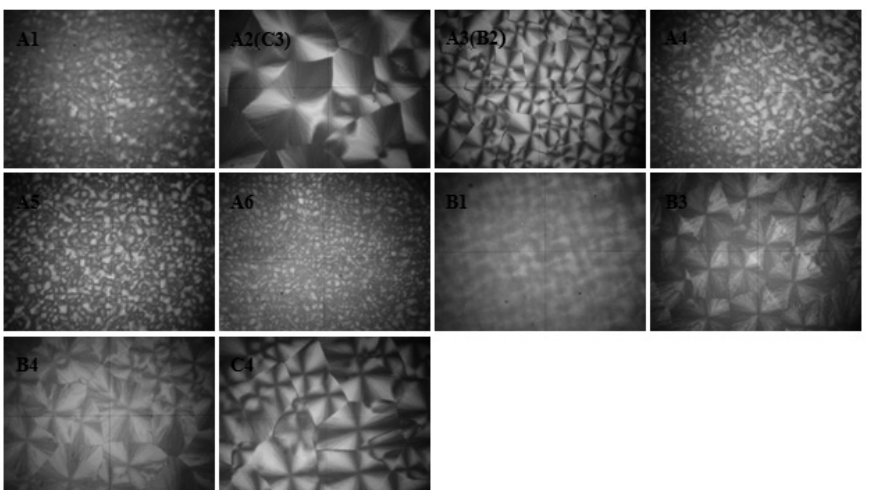

Figure 5. Optical micrographs (crossed polars: $\times 20$ ) of PMMA/PEG semiIPNs.

PMMA/PEG semi-IPNs are slightly opaque at room temperature, but they become transparent as soon as the temperature is increased above the melting point of PEG.
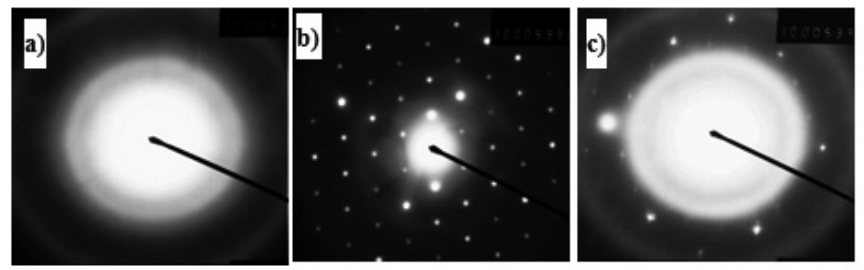

Figure 6. TEM diffraction photographs of a) PMMA networks, b) PEG1000 homopolymer and c) PMMA/35wt\% PEG1000 semi-IPNs.

The influence of molecular weight of PEG, content of PEG and crosslinking density on the crystalline morphology of PMMA/PEG in semi-IPNs was also studied by optical microscopy. Pure PEG is a semicrystalline polymer with spherulites structure, as shown in Figure 4. Here, we should mention that spherulitic texture is generally formed during the crystallization of polymer. The optical micrographs of PMMA/PEG semi-IPNs are presented in Figure 5. In contrast to pure PEG spherulites, crystal perfectness and crystal size of PEG in all semi-IPNs dramatically change; this means that the import of PMMA networks on PEG leads to obvious suppressing effect on the crystallization of PEG, although it unalters the spherulitic crystal structure. By comparing the micrograph of B2, B3, B4 with that of B1 in Figure 5, more addition of PEG to PMMA networks seems to lead to more complete crystallization of PEG; below $3.5 \mathrm{wt} \%$ of cross-linking density ( $\mathrm{C} 1$ and $\mathrm{C} 2)$, the PEG crystal can not be observed by optical microscopy, indicating PMMA/PEG semi-IPNs are fully amorphous; the cross-linking density clearly influences the crystallization behavior of PEG, as shown in Figure 5 (C3 and C4); the more the cross-linking density, the smaller the crystal of PEG. All above results agree with DSC 
discussion

Transmission electron microscopy (TEM) provided additional evidence that the crystalline structure existed in the PMMA/PEG semi-IPNs. It is known that PMMA network prepared by radical polymerization is amorphous, which shows an amorphous halo in Figure 6(a). PEG is a semicrystalline polymer with higher crystallinity and can show typical crystal diffraction pattern, corresponding to regular lattice structure, as show in Figure 6(b). Figure 6(c) is the PMMA $/ 35 \mathrm{wt} \%$ PEG1000 semi-IPNs diffraction pattern, testifying the semi-IPNs is composed of some amorphous regions and crystalline regions at the room temperature; it is believed that the crystalline PEG is separated from amorphous phase.

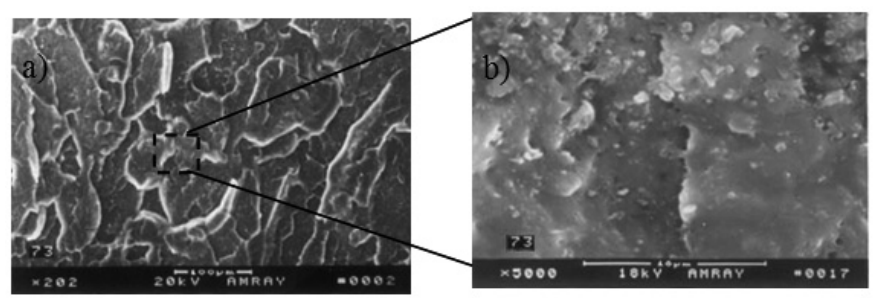

Figure 7. SEM images of fracture cross-section of A2 with different magnification: a) $\times 202$, b) $\times 5000$.

The microstructure of A2 was evaluated by SEM. Morphologies of low magnification of fracture cross-section are shown in Figure 7a). The fracture exhibits lamella-like structure and ductility, and the addition of PEG significantly changes the appearance of the impact fracture surface. SEM morphologies of high magnification of fracture cross-section are shown in Figure $7 \mathrm{~b}$ ). The exposed PEG crystals are observed because PMMA networks and PEG mix without strong chemical reaction. The interface of PMMA networks and PEG crystal phase can be easily distinguished. PEG crystal phase disperse homogeneously in the PMMA networks, but agglomeration occurs in the semi-IPNs involving higher PEG content.

\section{CONCLUSION}

The thermal behaviors and crystalline morphology properties of the PMMA/PEG semi-IPNs was studied by DSC, TEM and OM, respectively. The results indicated that the content of PEG and the cross-linking density of PMMA networks have an important influence on the phase separation in the PMMA/PEG semi-IPNs. When the content of PEG and cross-linking density is more than 35 and $3.5 \mathrm{wt} \%$, respectively, PMMA/PEG semi-IPNs are a mixture of PEG in the crystalline state with P(MMA-co-VP) networks in the amorphous state.

\section{REFERENCES AND NOTES}

1. J. Straka, P. Schmidt, J. Dybal, B. Schneider, J. Spevacek, Polymer 36, 1147 (1995).

2. X. Lu, R. A. Weiss, Macromolecules 25, 3242 (1992).

3. M. Lowman, N. A. Peppas, Polymer 41, 73 (2000).

4. S. Schantz, Macromolecules 30, 1419 (1997).

5. J. A. Zawada, C. M. Ylitalo, C. G. Fuller, R. H. Colby, T. E. Long, Macromolecules 25, 2896 (1992).

6. L. H. Sperling, V. Mishra, Polym. Advan. Technol. 7, 197 (1996).

7. S. J Park, J. S. Jin, J. Appl. Polym. Sci. 82, 775 (2001).

8. S. Desai, I. M. Thakore, Brennan, et a1., J. Appl, Polym. Sci. 83, 1576 (2002).

9. V. Athawale, S. Kolekar, J. Macromo.l Sci. Pur.e Appl. Chem. 37, 65 (2000).

10. A. Anzlovar, M. Zigon, Acta Chim. Slov. 52, 230 (2005).

11. F. Abbasi, H. Mirzadeh, J. Polym. Sci. B: Polym. Phys. 41, 2145 (2003).

12. F. Abbasi, H. Mirzadeh, A. A. Katbab, J. Appl. Polym. Sci. 85, 1825 (2002).

13. L. Liu, H. Sheardown, Biomaterials 26, 233 (2005).

14. M. S. Shin, S. J. Kim, I. Y. Kim, N. G. Kim, C. G. Song, S. I. Kim, J. Appl. Polym. Sci. 85, 957 (2002).

15. J. S. Turner, Y. L. Cheng, Macromolecules 36, 1962 (2003).
16. N. A. Chekina, V. N. Pavlyuchenko, V. F. Danilichev, N. A. Ushakov, S. A. Novikov, S. S. Ivanchev, Polym. Adv. Technol. 17, 872 (2006).

17. R.V. Kulkarni and B. Sa, Cur. Drug Del., 5, 256 (2008).

18. A. R. Kulkarni, K. S. Soppimath, T. M. Aminabhavi, and W. E. Rudzinski, Eur. J. Pharm. Biopharm., 51, 127 (2001).

19. A. P. Rokhade, S. A. Agnihotri, S. A. Patil, N. N. Mallikarjun,P. V. Kulkarni, and T. M. Aminabhavi, Carbohydrate Polym., 65, 243 (2006).

20. A. Anzlovar, M. Zigon, J. Polym. Sci. Part B: Polym. Phys 40, 115 (2002) $\square$

21. F. B. Calleja, E. G. Privalko, A. M. Fainleib, et a1, J. Macromol. Sci. Phys. 39, $131(2000)$.

22. Y. Chen, D. Ding, Z. Mao, Y. He, Y. Hu, W. Wu, X. Jiang, Biomacromolecules 9, 2609 (2008).

23. G. Liu, X. Ding, Y. Cao, Z. Zheng, Y. Peng, Macromolecules 37, 2228 (2004).

24. G. Liu, C. Guan, W. Zou, etc., J. Polym. Res. 14, 461 (2007).

25. G. Liu, Z. Liu, W. Zou, Z. Li, J. Peng, W. Cheng and S. Xu, Acta Chim. Slov. 56, 946 (2009).

26. G. Liu, X. Ding, Y. Cao, Z. Zheng, Y. Peng, Macromol. Rapid Commun. 26, 649 (2005).

27. S. W. Kuo, F. C. Chang, Macromolecules 34, 4089 (2001).

28. L. Hamon, Y. Grohens, A. Soldera, Y. Holl, Polymer 42, 9697 (2001). 